\title{
Aarno Hohti
}

On supercomplete uniform spaces. II

Czechoslovak Mathematical Journal, Vol. 37 (1987), No. 3, 376-385

Persistent URL: http://dml.cz/dmlcz/102165

\section{Terms of use:}

(C) Institute of Mathematics AS CR, 1987

Institute of Mathematics of the Czech Academy of Sciences provides access to digitized documents strictly for personal use. Each copy of any part of this document must contain these Terms of use.

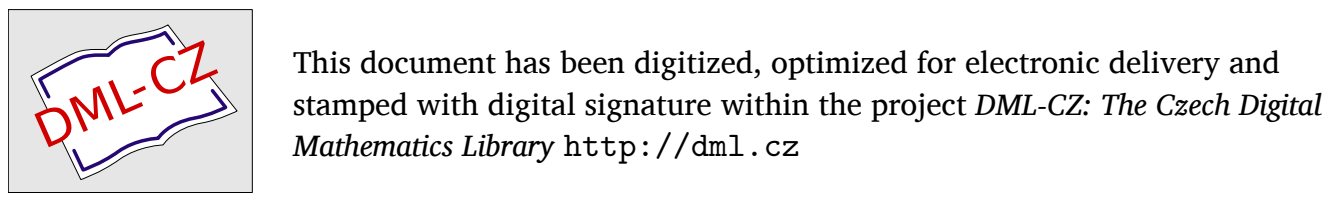




\title{
ON SUPERCOMPLETE UNIFORM SPACES II
}

\author{
AARNo HoHTI, Helsinki \\ (Received September 21; 1983; in revised form April 20, 1985)
}

1. Introduction. We proved in [9] that a uniform space $\mu X$ is supercomplete if and only if the locally fine coreflection of $\mu X \times \mathscr{F} \beta X$ is equinormal. Here we shall continue this work by characterizing the supercomplete $p$-spaces whose product with every supercomplete space is supercomplete. In the spirit of Telgársky [20] we show the importance of $C$-scattered spaces in this context. The results differ from the corresponding results for paracompact spaces (being obtained by [16] and [20]) and the role of $p$-spaces is different since supercompleteness is not preserved under uniformly continuous, perfect onto maps.

The study of completeness in uniform hyperspaces is facilitated by Isbell's theorem [12]: the hyperspace $H(\mu X)$ of a uniform space $\mu X$ is complete if and only if $X$ is topologically paracompact and the Ginsberg-Isbell locally fine coreflection ([7]) $\lambda \mu X$ is fine. (The result is to be contrasted with the result of [17], improving [22], which states that if $\mu X$ is complete then so is the uniform hyperspace $K(\mu X)$ of all compact subsets of $X$. See also [2] and [10] for a short proof.)

In order to apply the locally fine coreflection here, we need some preliminary definitions. Let $\mu$ and $v$ be filters of coverings of a set $X$, ordered by the relation of refinement. Then $v / \mu$ denotes the family of all covers of $X$ having a refinement of the form $\left\{U_{i} \cap V_{j}^{i}\right\}$, where $\left\{U_{i}\right\} \in \mu$ and for each $i,\left\{V_{j}^{i}\right\} \in v$. The successive derivatives $\mu^{(\alpha)}$ are defined by setting $\mu^{(0)}=\mu, \mu^{(\alpha+1)}=\mu^{(\alpha)} \mid \mu$ and $\mu^{(\beta)}=\bigcup\left\{\mu^{(\alpha)}: \alpha<\beta\right\}$ if $\beta$ is a limit ordinal. There is the least ordinal $\alpha$ such that $\mu^{(\alpha+1)}=\mu^{(\alpha)}$ and for which we define $\lambda \mu=\mu^{(\alpha)}$. These derivatives can be used instead of the original GinsburgIsbell derivatives as explained in [9]. The fine uniformity of $X$ (resp. the fine uniform space associated with $X)$ will be denoted by $\mathscr{F}(X)($ resp. $\mathscr{F} X)$ ). If $X$ and $Y$ are completely regular spaces and the locally fine coreflection of $\mathscr{F}(X) \times \mathscr{F}(Y)$ is fine, i.e. $\lambda(\mathscr{F}(X) \times \mathscr{F}(Y))=\mathscr{F}(X \times Y)$, then we say that $X \times Y$ has the $\lambda$-property. (Strictly speaking the $\lambda$-property is a property of the pair $(X, Y)$.) The completion of a uniform space $\mu X$ will be denoted by $\pi \mu X$. For a family $\mathscr{V}$ of subsets of $X, \mathscr{V} \uparrow A=$ $=\{V \cap A: V \in \mathscr{V}\}$ denotes the restriction of $\mathscr{V}$ to a subset $A$ of $X$. Similarly if $\mu$ is a family of covers of $X$ (e.g. a uniformity), then $\mu\lceil A=\{\mathscr{U} \uparrow A$ : $\mathscr{U} \in \mu\}$ denotes 
the restriction of $\mu$ to $A$. Recall that a subspace $A$ of a topological space $X$ is called $P$-embedded [19] if every continuous pseudometric on $A$ can be extended to a continuous pseudometric on $X$, or, equivalently, if $\mathscr{F}(A)=\mathscr{F}(X) \mid A$. A closed subspace of a collectionwise normal space is $P$-embedded [19]. Paracompact $p$-spaces were introduced in [1]. Recall that $X$ is a paracompact $p$-space if and only if there is a metrizable space $Y$ and a perfect map of $Y$ onto $X$. ([1], Theorem 16). Finally, a topological space $X$ is called $C$-scattered [20] if every nonempty closed subspace $F$ of $X$ contains a point with a compact neighbourhood in $F$.

2. The product of a fine paracompact $C$-scattered space with a fine paracompact space. We have shown in [8] that for every completely regular space $X$ and every compact space $K$ the product $X \times K$ has the $\lambda$-property. Here we shall extend the result to the case where $K$ is replaced by a $C$-scattered space, provided that the factors are paracompact. The result would follow quicker by using results in [14] and [15], but the proof would be based on a rather non-elementary notion of the hypercompletion of a locale and thus we give a completely elementary argument for the general reader's benefit. The technique of exhaustion has been used previously by J. R. Isbell.

Theorem 2.1. Let $X$ be a $C$-scattered paracompact space and let $Y$ be a paracompact space. Then $X \times Y$ has the $\lambda$-property.

Proof. We shall show that every open cover of $X \times Y$ belongs to $\lambda(\mathscr{F}(X) \times \mathscr{F}(Y))$. It then follows that $\lambda(\mathscr{F}(X) \times \mathscr{F}(Y))=\mathscr{F}(X \times Y)$. We will define a sequence of pairs $\left\langle W_{\alpha}, S_{\alpha}\right\rangle$, where $S_{\alpha}$ is a closed subset of $X$ and $W_{\alpha}$ is an open subset of $X$ such that $\bar{W}_{\alpha} \cap S_{\alpha}$ is compact.

Step 0 . As $X$ is $C$-scattered, there is a point $x_{0} \in X$ and an open neighbourhood $W_{0}$ of $x_{0}$ such that $\bar{W}_{0}$ is compact. Let $S_{0}=X$.

Step $\alpha$. Suppose that $\left\langle W_{\beta}, S_{\beta}\right\rangle$ has been defined for all $\beta<\alpha$ and let $S_{\alpha}=$ $=X \backslash\left\{W_{\beta}: \beta<\alpha\right\}$. Then $S_{\alpha}$ is a closed subset of $X$. If $S_{\alpha}=\emptyset$, then the inductive definition stops here. Otherwise there is a point $x_{\alpha} \in S_{\alpha}$ and an open neighbourhood $W_{\alpha}$ of $x_{\alpha}$ in $X$ such that $\bar{W}_{\alpha} \cap S_{\alpha}$ is compact.

Let $\alpha$ be the least ordinal such that $X=\bigcup\left\{W_{\beta}: \beta<\alpha\right\}$. Then $\left\{\left\langle W_{\beta}, S_{\beta}\right\rangle: \beta<\alpha\right\}$ is called an exhaustion of length $\alpha$. Let $P(\alpha)$ be the following statement:

"If $Z_{1}$ is a $C$-scattered paracompact space, $Z_{2}$ is a paracompact space and $Z_{1}$ has an exhaustion of length $\leqq \alpha$, then every open cover of $Z_{1} \times Z_{2}$ belongs to $\lambda\left(\mathscr{F}\left(Z_{1}\right) \times \mathscr{F}\left(Z_{2}\right)\right) . "$

If $X$ has an exhaustion of length 1 , then $X$ is compact and it follows from 5.1.8. in [8] that $P(1)$ is valid. Assume that $P(\beta)$ is valid whenever $\beta<\alpha$ suppose that $X$ has an exhaustion $\left\{\left\langle W_{\beta}, S_{\beta}\right\rangle: \beta<\alpha\right\}$ of length $\alpha$ and let $\mathscr{G}$ be an open cover of $X \times Y$. We shall consider two cases.

Case 1. $\alpha=\beta+1$. Then $X \backslash\left\{W_{\gamma}: \gamma<\beta\right\} \subset W_{\beta}$ and hence $\bar{S}_{\beta}=S_{\beta}$ is compact. Let $y \in Y$. For each $x \in S_{\beta}$ choose an open neighbourhood $U_{x, y}$ of $x$ and an open neighbourhood $V_{x, y}$ of $y$ such that $U_{x, y} \times \bar{V}_{x, y} \subset G$ for some $G \in \mathscr{G}$. As $S_{\beta}$ is compact, we can find a finite subset $F_{y} \subset S_{\beta}$ such that $S_{\beta} \subset \bigcap\left\{U_{x, y}: x \in F_{y}\right\}$. Define $V_{y}=$ 
$\left.=\bigcap V_{x, y}: x \in F_{y}\right\}$. Then $V_{y}$ is an open neighbourhood of $y$ and for each $x \in F_{y}$ there is a $G_{x, y} \in \mathscr{G}$ such that $U_{x, y} \times \bar{V}_{y} \subset G_{x, y}$. Now $\mathscr{V}=\left\{V_{y}: y \in Y\right\}$ is an open cover of $Y$ and thus $\mathscr{V} \in \mathscr{F}(Y)$ since every open cover of a paracompact space is normal. As $S_{\beta}$ is closed, there exists an open subset $O_{y}$ of $X$ such that

$$
S_{\beta} \subset O_{y} \subset \bar{O}_{y} \subset \bigcap\left\{U_{x, y}: x \in F_{y}\right\} .
$$

Now $Z_{y}=X \backslash O_{y}$ is a closed subspace of $X$ and clearly $Z_{y}$ has an exhaustion of length $\leqq \beta$. By the induction hypothesis, every open cover of $Z_{y} \times \bar{V}_{y}$ belongs to $\lambda\left(\mathscr{F}\left(Z_{y}\right) \times \mathscr{F}\left(\bar{V}_{y}\right)\right)$. Therefore

$$
\mathscr{G} \uparrow\left(Z_{y} \times \bar{V}_{y}\right) \in \lambda\left(\mathscr{F}\left(Z_{y}\right) \times \mathscr{F}\left(\bar{V}_{y}\right)\right) .
$$

The closed subspaces $Z_{y}$ and $\bar{V}_{y}$ are $P$-embedded, whence

$$
\mathscr{F}\left(Z_{y}\right) \times \mathscr{F}\left(\bar{V}_{y}\right)=\left(\mathscr{F}(X) \mid Z_{y}\right) \times\left(\mathscr{F}(Y) \mid \bar{V}_{y}\right)=(\mathscr{F}(X) \times \mathscr{F}(Y)) \mid\left(Z_{y} \times \bar{V}_{y}\right)
$$

by the definition of the product uniformity. On the hand, $\lambda$ preserves subspaces and it follows from the preceding two formulas that

$$
\mathscr{G} \uparrow\left(Z_{y} \times \bar{V}_{y}\right) \in[\lambda(\mathscr{F}(X) \times \mathscr{F}(Y))] \uparrow\left(Z_{y} \times \bar{V}_{y}\right) .
$$

Now $\mathscr{H}_{y}=\left\{X \backslash O_{y}\right\} \cup\left\{U_{x, y}: x \in F_{y}\right\}$ is an open cover of $X$. As $X$ is paracompact, $\mathscr{H}_{y}$ belongs to $\mathscr{F}(X)$. By the above, the restriction of $\mathscr{G}$ to any element $H \times \bar{V}_{y}$, where $H \in \mathscr{H}_{y}$, is a " $\lambda$-uniform" cover of $H \times \bar{V}_{y}$ relative to $\mathscr{F}(X) \times \mathscr{F}(Y)$. (Recall that $\left.U_{x, y} \times \bar{V}_{y} \subset G_{x, y} !\right)$ As $\left\{H \times \bar{V}_{y}: H \in \mathscr{H}\right\}$ is a uniform cover of $X \times \bar{V}_{y}$ with respect to $\mathscr{F}(X) \times \mathscr{F}(Y)$ (because $\vec{\nabla}_{y}$ is $P$-embedded) it follows that the restriction of $\mathscr{G}$ to $X \times \bar{V}_{y}$ is a $\lambda$-uniform cover. But $\mathscr{V} \in \mathscr{F}(Y)$ and therefore $\left\{X \times \bar{V}_{y}: y \in Y\right\}$ is a uniform cover of $X \times Y$; as a consequence of the definition of $\lambda$ via the successive derivatives, $\mathscr{G}$ is a $\lambda$-uniform cover $X \times Y$ relative to $\mathscr{F}(X) \times \mathscr{F}(Y)$, i.e. $\mathscr{G} \in$ $\in \lambda(\mathscr{F}(X) \times \mathscr{F}(Y))$.

Case $2 . \alpha$ is a limit ordinal. Then $X=\bigcup\left\{W_{\beta}: \beta<\alpha\right\}$ and each $\bar{W}_{\beta}$ has an exhaustion of length $\leqq \beta+1$. Thus, by the induction hypothesis, for each $\beta<\alpha$

$$
\mathscr{G} \uparrow\left(\bar{W}_{\beta} \times Y\right) \in[\lambda(\mathscr{F}(X) \times \mathscr{F}(Y))] \uparrow\left(\bar{W}_{\beta} \times Y\right)
$$

because $\bar{W}_{\beta}$ is $P$-embedded. As $X$ is paracompact, $\left\{W_{\beta}: \beta<\alpha\right\} \in \mathscr{F}(X)$. It follows that $\mathscr{G}$ belongs to $\lambda(\mathscr{F}(X) \times \mathscr{F}(Y))$. This completes our proof.

Remark. R. Telgársky proved in [20] that if $X$ is a $C$-scattered paracompact space and $Y$ is a paracompact space, then $X \times Y$ is paracompact. His result follows from the proof above. Moreover, if $X$ has a countable cover by closed $C$-scattered subspaces and $Y$ is paracompact, then $X \times Y$ is paracompact. As we shall see later in this paper, the latter statement cannot be extended to the case of $\lambda$-property.

Corollary 2.2. Let $\mu X$ be a $C$-scattered supercomplete space and let $\nu Y$ be a supercomplete space. Then $\mu X \times \nu Y$ is supercomplete.

Proof. As $\mu X$ and $\nu Y$ are supercomplete, it follows from Isbell's theorem that $X$ and $Y$ are paracompact, $\lambda \mu X=\mathscr{F} X$ and $\lambda \nu Y=\mathscr{F} Y$. By Theorem 2.1, $\lambda(\mathscr{F}(X) \times$ 
$\times \mathscr{F}(Y))=\mathscr{F}(X \times Y)$. Thus, $\lambda(\mu X \times \nu Y)=\lambda(\lambda \mu X \times \lambda \nu Y)=\lambda(\mathscr{F}(X) \times \mathscr{F}(Y))=$ $=\mathscr{F}(X \times Y)$, as required. Moreover, $X \times Y$ is paracompact, by [20].

3. Some lemmas. It was stated in Exercise VII 8(e) of [13] that a fine separable metrizable space $X$ has the property that $X \times Y$ is supercomplete for every fine separable metrizable space $Y$ iff $X$ is locally compact. However, the statement is not valid as can be seen from Theorem 2.1. Anyhow, the claim becomes true if the words "locally compact" are replaced by the word " $C$-scattered". As a matter of fact, a corresponding statement is true in the class of paracompact $p$-spaces. To prove this we shall need a generalized version of Exercise VII 8(d) in [13]. First we will establish three lemmas. A map $f: \mu X \rightarrow \nu Y$ is called $\lambda$-uniformly continuous if $f: \lambda \mu X \rightarrow v Y$ is uniformly continuous. A product of $\lambda$-uniformly continuous maps is $\lambda$-uniformly continuous.

Lemma 3.1. Let $f: \mu X \rightarrow \nu Y$ be $\lambda$-uniformly continuous. Then $f^{-1}(\mathscr{U}) \in \lambda \mu$ for each $\mathscr{U} \in \lambda v$.

Proof. The lemma can be proved by a straightforward induction.

Lemma 3.2. Let $\mu X$ and $v Y$ be uniform spaces, let $f: \mu X \rightarrow v Y$ be a $\lambda$-uniformly continuous perfect onto map and let $v Y$ be supercomplete. Then $\mu X$ is supercomplete.

Proof. Let $\mathscr{V}$ be an open cover of $X$. We must show that $\mathscr{V} \in \lambda \mu$. For each $y \in Y$ there is an open uniform cover $\mathscr{U}_{y} \in \mu$ such that $\mathscr{V}\left\lceil\operatorname{St}\left(f^{-1}\{y\}, \mathscr{U}_{y}\right)\right.$ is a uniform cover, since the point-inverse $f^{-1}\{y\}$ is compact. Note that

$$
y \in Y-f\left[X-\operatorname{St}\left(f^{-1}\{y\}, \mathscr{U}_{y}\right]=W_{y},\right.
$$

$W_{y}$ is an open neighbourhood of $y$ and that $f^{-1}\left[W_{y}\right] \subset \operatorname{St}\left(f^{-1}\{y\}, \mathscr{U}_{y}\right)$. Now $\mathscr{W}=\left\{W_{y}: y \in Y\right\}$ is an open cover of $Y$ and consequently $\mathscr{W} \in \lambda v$ since $v Y$ is supercomplete. By the previous lemma, $f^{-1}(\mathscr{W}) \in \lambda \mu$. But

$$
f^{-1}(\mathscr{W})<\left\{\operatorname{St}\left(f^{-1}\{y\}, \mathscr{U}_{y}\right): y \in Y\right\}
$$

and therefore for each $W \in \mathscr{W}, \mathscr{V}\left\lceil f^{-1}[W]\right.$ is a uniform cover of $f^{-1}[W]$. It follows that $\mathscr{V} \in \lambda \mu$, as desired.

Recall that a completely regular space $X$ is Čech-complete if $X$ is a $G_{\delta}$-subspace of $\beta X$. Z. Frolík proved in [5] that $X$ is Čech-complete and paracompact iff there is a complete metric space $Y$ and a perfect onto map $f: X \rightarrow Y$.

Corollary 3.3. The product of a countable family of Čech-complete supercomplete spaces is supercomplete.

Proof. Let $\left\{\mu_{n} X_{n}\right\}$ be a sequence of Čech-complete supercomplete spaces. For each $n$ there exists a complete metric space $Y_{n}$ and a perfect onto map $f_{n}: X_{n} \rightarrow Y_{n}$. As $\mu_{n} X_{n}$ is supercomplete, $\lambda \mu_{n} X_{n}=\mathscr{F} X_{n}$. It is well known that $f_{n}: \mathscr{F} X_{n} \rightarrow Y_{n}$ is uniformly continuous - consequently each $f_{n}$ is $\lambda$-uniformly continuous. Let $\mu X=$ $=\Pi\left\{\mu_{n} X_{n}\right\}$ and let $Y=\Pi\left\{Y_{n}\right\}$. The product $\Pi\left\{f_{n}\right\}$ is perfect ([5]) and $\lambda$-uniformly 
continuous. As $Y$ is a complete metric space, it follows from Lemma 3.2. that $\mu X$ is supercomplete. (See also [7], 4.2.)

Remark. It was shown by Frolik [5] that the product of a countable family of Čech-complete paracompact spaces is paracompact.

Lemma 3.4. Let $X$ and $Y$ be completely regular spaces such that $X \times Y$ is Lindelöf. An open cover $\mathscr{G}$ of $X \times Y$ belongs to $\lambda(\mathscr{F}(X) \times \mathscr{F}(Y))$ if and only if there exist Cech-complete paracompact subspaces $M \subset \beta X, N \subset \beta Y$ such that $X \subset M$, $Y \subset N$ and $\mathscr{G}$ can be extended to an open cover of $M \times N$.

Proof. To prove necessity we shall proceed by induction. Hence let $\mathscr{G} \in \lambda(\mathscr{F}(X) \times$ $\times \mathscr{F}(Y))$. Then there is an $\alpha$ such that $\mathscr{G} \in(\mathscr{F}(X) \times \mathscr{F}(Y))^{(\alpha)}$. To start with, let $\alpha=0$. Then $\mathscr{G}$ is a uniform cover and thus there exist $\mathscr{U} \in \mathscr{F}(X)$ and $\mathscr{V} \in \mathscr{F}(Y)$ such that $\mathscr{U} \times \mathscr{V}<\mathscr{G}$. We can find continuous pseudometrics $\varrho$ and $\sigma$ on $X$ and $Y$, respectively, such that $\mathscr{U}$ (resp. $\mathscr{V}$ ) is a uniform cover of $\varrho X$ (resp. $\sigma Y$ ). Let $Z_{1}$ and $Z_{2}$ be the corresponding natural metric quotients and let $g: \varrho X \rightarrow Z_{1}, h: \sigma Y \rightarrow Z_{2}$ be the quotient maps. Let $i: Z_{1} \rightarrow \pi Z_{1}$ and $j: Z_{2} \rightarrow \pi Z_{2}$ be the natural embeddings. Put $\phi_{1}=i \circ g, \phi_{2}=j \circ h$. Then $\mathscr{G}^{\prime}=\left(\phi_{1} \times \phi_{2}\right)(\mathscr{G})$ is a uniform open cover of the subspace $Z_{1} \times Z_{2}$ of $\pi\left(Z_{1} \times Z_{2}\right)$ and therefore $([13]) \mathscr{G}^{\prime}$ can be extended to an open (uniform) cover $\mathscr{H}$ of $\pi Z_{1} \times \pi Z_{2}$. Let $e_{1}: \pi Z_{1} \rightarrow \beta \pi Z_{1}$ and $e_{2}: \pi Z_{2} \rightarrow \beta \pi Z_{2}$ be the natural embeddings and let $\psi_{1}: \beta X \rightarrow \beta \pi Z_{1}, \psi_{2}: \beta Y \rightarrow \beta \pi Z_{2}$ be the Stoneextensions of $e_{1} \circ \phi_{1} \circ$ id and $e_{2} \circ \phi_{2} \circ$ id, respectively, ([21], p. 9). The situation can be described by the following commutative diagram.

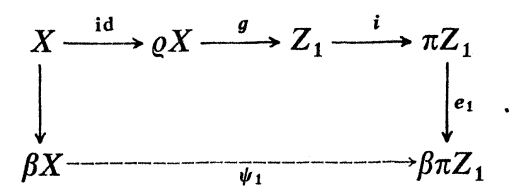

Then $\psi_{1}$ and $\psi_{2}$ are perfect onto maps and hence $M=\psi_{1}^{-1}\left[\pi Z_{1}\right]$ and $N=\psi_{2}^{-1}\left[\pi Z_{2}\right]$ are Čech-complete and paracompact. Now $\left(\psi_{1} \times \psi_{2}\right)^{-1}(\mathscr{H})$ is an extension of $\mathscr{G}$ to an open cover of $M \times N$,

Thus, suppose that the claim is valid whenever $\beta<\alpha$. We can assume that $\alpha$ is a successor ordinal, say $\alpha=\beta+1$. Then there is an open uniform cover $\left\{U_{s}: s \in S\right\}-$ where we can assume that $S$ is countable - of $\mathscr{F} X \times \mathscr{F} Y$ such that for each $s \in S$, $\mathscr{G}\left\lceil U_{s}\right.$ has a refinement $\left\{U_{s} \cap V_{t}^{s}\right\}$, where $\left\{V_{t}^{s}\right\} \in(\mathscr{F}(X) \times \mathscr{F}(Y))^{(\beta)}$ is open. By the inductive hypothesis, there exist Čech-complete paracompact subspaces $M, M_{s} \subset$ $\subset \beta X, N, N_{s} \subset \beta Y$ such that $\left\{U_{s}\right\}$ can be extended over $M \times N$ and for each $s \in S$, $\left\{V_{t}^{s}\right\}$ can be extended over $M_{s} \times N_{s}$. Define $M^{\prime}=\bigcap\left\{M_{s}: s \in S\right\} \cap M$ and $N^{\prime}=$ $=\bigcap\left\{N_{s}: s \in S\right\} \cap N$. Hence $\mathscr{G}$ has an open refinement which can be extended over $M^{\prime} \times N^{\prime}$. Let $\mathscr{W}$ be an extension of $\left\{U_{s} \cap V_{t}^{s}\right\}$ to an open cover of $M^{\prime} \times N^{\prime}$. For each $G \in \mathscr{G}$ choose an open subset $G^{\prime}$ of $M^{\prime} \times N^{\prime}$ such that $G=G^{\prime} \cap(X \times Y)$ and define $G^{\prime \prime}=\bigcup\{W \in \mathscr{W}: W \cap(X \times Y) \subset G\} \cup G^{\prime}$. Then $\left\{G^{\prime \prime}: G \in \mathscr{G}\right\}$ is the desired extension of $\mathscr{G}$ to an open cover of $M^{\prime} \times N^{\prime}$. Furthermore, $M^{\prime}$ and $N^{\prime}$ are Čech- 
complete and paracompact since the property of being Čech-complete and paracompact is preserved in countable intersections. (For, this property is preserved in countable products and hence in limits of inverse sequences.)

For sufficiency, let $\mathscr{G}$ be an open cover of $X \times Y$ and let $M \subset \beta X, N \subset \beta Y$ be Čech-complete paracompact subspaces such that $X \subset M, Y \subset N$ and $\mathscr{G}$ has an extension to an open cover $\widetilde{G}$ of $M \times N$. Now $\mathscr{F} M \times \mathscr{F} N$ is supercomplete by Corollary 3.3. Thus, $\widetilde{G} \in \mathscr{F}(M \times N)=\lambda(\mathscr{F}(M)) \times \mathscr{F}(N))$. As $\lambda$ preserves subspaces, $\mathscr{G} \in \lambda((\mathscr{F}(M) \times \mathscr{F}(N))\lceil(X \times Y)=\lambda[(\mathscr{F}(M) \times \mathscr{F}(N))\lceil(X \times Y)]=$ $=\lambda[(\mathscr{F}(M)\lceil X) \times(\mathscr{F}(N)\lceil Y)] \subset \lambda(\mathscr{F}(X) \times \mathscr{F}(Y))$ and our proof is complete.

We shall use Lemma 3.4. in the form of the following corollary.

Corollary 3.5. Let $X$ and $Y$ be completely regular spaces such that $X \times Y$ is Lindelof. Then $\mathscr{F} X \times \mathscr{F} Y$ is supercomplete if and only if for each compact $K \subset(\beta X \times \beta Y)-(X \times Y)$ there exist Čech-complete paracompact subspaces $M \subset \beta X, N \subset \beta Y$ such that $X \subset M, Y \subset N$ and $(M \times N) \cap K=\emptyset$.

Proof. For necessity, suppose that $\mathscr{F} X \times \mathscr{F} Y$ is supercomplete. Then $X \times Y$ has the $\lambda$-property. Given a compact set $K \subset(\beta X \times \beta Y)-(X \times Y)$, choose for each $(x, y) \in X \times Y$ an open neighbourhood $U_{x} \times V_{y}$ of $(x, y)$ in $\beta X \times \beta Y$ such that $\left(\operatorname{cl}_{\beta X} U_{x} \times \operatorname{cl}_{\beta Y} V_{y}\right) \cap K=\emptyset$. Then $\mathscr{G}=\left\{\left(U_{x} \cap X\right) \times\left(V_{y} \cap Y\right):(x, y) \in X \times Y\right\}$ is an open cover of $X \times Y$ and hence by 3.4 there exist Čech-complete paracompact $M \subset \beta X, N \subset \beta Y$ such that $X \subset M, Y \subset N$ and $\mathscr{G}$ has an extension $\widetilde{G}$ to an open cover of $M \times N$. Let $G \in \widetilde{G}$ and choose $x \in X, y \in Y$ such that $G \cap(X \times Y)=$ $=\left(U_{x} \cap X\right) \times\left(V_{y} \cap Y\right)$. As $X \times Y$ is dense in $\beta X \times \beta Y$,

$$
G \subset \operatorname{cl}_{(\beta X \times \beta Y)}(G \cap(X \times Y))=\operatorname{cl}_{\beta X} U_{x} \times \operatorname{cl}_{\beta Y} V_{y} \subset(\beta X \times \beta Y)-K .
$$

Thus, $(M \times N) \cap K=\emptyset$.

For sufficiency, we use 3.4 to show that every open cover of $X \times Y$ belongs to $\lambda(\mathscr{F}(X) \times \mathscr{F}(Y))$. If $\mathscr{G}$ is an open cover of $X \times Y$, then $\mathscr{G}$ can be extended to a family $\mathscr{G}^{\prime}$ of open subsets of $\beta X \times \beta Y$. Let $K=(\beta X \times \beta Y)-U\left(\mathscr{G}^{\prime}\right)$. By assumption there exist Čech-complete paracompact $M \subset \beta X, N \subset \beta Y$ such that $X \subset M, Y \subset N$ and $(M \times N) \cap K=\emptyset$. It follows that $\mathscr{G}^{\prime}\lceil(M \times N)$ is an extension of $\mathscr{G}$ to an open cover of $M \times N$.

Finally, we shall need a lemma that enables us to move from paracompact $p$-spaces to separable metrizable spaces.

Lemma 3.6. Let $X$ be a metrizable space which is not $C$-scattered. Then $X$ contains a separable closed subspace which is not $C$-scattered.

Proof. As $X$ is not $C$-scattered, there is a closed subspace $F \subset X$ which is nowhere locally compact in itself. For each $x \in F$, let $\left\{B_{n, x}\right\}$ be a countable base of closed neighbourhoods of $x$ in $F$. For all $x \in F$ and $n<\omega$, the set $B_{n, x}$ is not compact and thus there is a closed, discrete countably infinite subset $S_{n, x_{i}} \subset B_{n, x}$. Let $x_{0} \in F$ be arbitrary. Define $Y_{0}=\bigcup\left\{S_{n, x_{0}}: n<\omega\right\}$. Assume that $Y_{m}$ has been defined and let $Y_{m+1}=\bigcup\left\{S_{n, x}: x \in Y_{m}\right\} \cup Y_{m}$. Finally, let $Y=\bar{U}\left\{Y_{m}: m<\omega\right\}$. Then $Y$ is the 
closure of a countable subset of $F$ and hence $Y$ is separable. Suppose that $y \in Y$ and let $U$ be a neighbourhood of $y$. There is an $m<\omega$, an $x \in Y_{m-1}$ and $n<\omega$ such that $B_{n, x} \subset U$. Thus, $S_{n, x} \subset U$ and hence $U$ is not compact. As a consequence, $Y$ is nowhere locally compact.

4. A partial converse of 2.1. Paracompact $p$-spaces were introduced in [1]. Recall that $X$ is a paracompact $p$-space if and only if there is a metrizable space $Y$ and a perfect onto map $f: X \rightarrow Y$. ([1], Theorem 16.)

Theorem 4.1. Let $X$ be a paracompact p-space which is not $C$-scattered. Then there is a separable metrizable space $Y$ such that $\mathscr{F} X \times \mathscr{F} Y$ is not supercomplete.

Proof. There is a metrizable space $Z$ and a perfect onto map $f: X \rightarrow Z$. It was proved in [17] that the class of $C$-scattered spaces is perfect (i.e.[17] the images and pre-images of $C$-scattered spaces under perfect maps are $C$-scattered). It follows that $Z$ is not $C$-scattered. By Lemma $3.6 Z$ contains a closed separable subspace $F$ which is not $C$-scattered and we can assume that $F$ is nowhere locally compact in itself. Let $\widetilde{F}$ be a metrizable compactification of $F$. The remainder $\widetilde{F}-F$ is dense in $\widetilde{F}$. (For, otherwise $\widetilde{F}$ would contain an open subset whose closure in $\widetilde{F}$ would be contained in $F$.) Put $E=f^{-1}[F]$ and note that $f \nmid E$ is a perfect map. Let $g: \beta E \rightarrow \tilde{F}$ be the Stone-extension of $f\lceil E$. Now $\widetilde{F}$ is a compactification of $\widetilde{F}-F$ and hence there exists a quotient map $h: \beta(\widetilde{F}-F) \rightarrow \widetilde{F}$ that is the identity on $\widetilde{F}-F$. (Let $h$ be the Stone-extension of the identity.) Let $\Delta(\widetilde{F})$ be the diagonal of $\widetilde{F} \times \widetilde{F}$ and define $K=(g \times h)^{-1}[\Delta(\widetilde{F})]$. Then $K$ is a compact subset of $\beta E \times \beta(\widetilde{F}-F)$ such that

$$
K \subset(\beta E \times \beta(\tilde{F}-F))-(E \times(\tilde{F}-F)) .
$$

Indeed, suppose that $(p, q) \in K$. Then

$$
(g(p), h(q)) \in \Delta(\widetilde{F}) \subset(\widetilde{F} \times \widetilde{F})-(F \times(\widetilde{F}-F)) .
$$

Hence, if $p \in E$ and $q \in \widetilde{F}-F$, we would have $(g(p), h(q))=(g(p), q) \in F \times$ $\times(\tilde{F}-F)$, which is impossible.

First we shall show that $\mathscr{F} E \times \mathscr{F}(\widetilde{F}-F)$ is not supercomplete. To obtain a contradiction, suppose that $\mathscr{F} E \times \mathscr{F}(\widetilde{F}-F)$ is supercomplete. As $E \times(\widetilde{F}=F)$, being a product of two Lindelöf $p$-spaces is Lindelöf, it follows from Corollary 3.5. that there exist Čech-complete spaces $M \subset \beta E, N \subset \beta(\widetilde{F}-F)$ such that $E \subset M$, $\widetilde{F}-F \subset N$ and $(M \times N) \cap K=\emptyset$. Thus, $(g[M] \times h[N]) \cap \Delta(\widetilde{F})=\emptyset$ from which it follows that $g[M] \cap h[N]=\emptyset$. As $h$ keeps $\widetilde{F}-F$ fixed, we have $\widetilde{F}-F \subset h[N]$. On the other hand, $g$ extends $f \nmid E$ and hence $F \subset g[M]$. Now $F \cap h[N]=\emptyset$, for otherwise $g[M] \cap h[N] \neq \emptyset$. Thus, $h[N]=\widetilde{F}-F$. It follows that also $g[M]=$ $=F$. Now $f \nmid E$ is perfect and hence $g[\beta E-E] \subset \tilde{F}-F([21]$, p. 275). Thus, $M \subset g^{-1}[F] \subset E$ and consequently $M=E$. Similarly $N=\widetilde{F}-F$. It follows that $E$ is Čech-complete and therefore so is $F$. Thus, both $F$ and $\widetilde{F}-F$ are Čechcomplete. Hence, both $F$ and $\widetilde{F}-F$ are $F_{\sigma}$-subsets of $\widetilde{F}$. As a consequence, they are of the first category in $\widetilde{F}$. But then $\widetilde{F}$ is of the first category which contradicts the 
Baire Category Theorem. Thus, $\mathscr{F} E \times \mathscr{F}(\tilde{F}-F)$ is not supercomplete. Put $Y=\widetilde{F}-F$. As $\lambda$ preserves subspaces, every closed subspace of a supercomplete space is supercomplete. It follows that $\mathscr{F} X \times \mathscr{F} Y$ is not supercomplete, as required.

Corollary 4.2. Let $\mu X$ be a supercomplete p-space. Then the following statements are equivalent:

i) $H(\mu X \times v Y)$ is complete for every p-space $v Y$ such that $H(v Y)$ is complete;

ii) $X$ is $C$-scattered.

\section{Concluding remarks.}

Remark 1. Call a uniform space $\mu X \sigma$-discretely refinable, if every open cover of $X$ has a $\sigma$-uniformly discrete refinement (i.e. a refinement which is a countable union of $\mu$-uniformly discrete subfamilies). The $\delta$-discretely refinable spaces have been studied by Frolík [6] under the title "paracompact uniform spaces". (They are originally defined in [4].) There was a problem whether every complete $\sigma$-discretely refinable uniform space is supercomplete. Here we see (by examining the proof of 4.1.) that for example the product space $\mathscr{F} J \times \mathscr{F} Q$, where $J$ is the space of irrationals and $Q$ denotes the space of rationals, is a complete Lindelöf space which is not supercomplete. Actually, we can use the idea behind the proof of 4.1. to show that $\mathscr{F} Q \times$ $\times \mathscr{F} Q$ is not supercomplete. It is enough to note that $(I \times I)-[(Q \cap I) \times(Q \cap I)]$ contains the sphere with the radius $1 / 4$ and center $(a, 1 / 2)$, where $a \in] 1 / 4,3 / 4[$ is transcendental. It easily follows from 3.5. that $\mathscr{F}(Q \cap I) \times \mathscr{F}(Q \cap I)$ is not supercomplete. Let $X$ be a separable metrizable space and suppose that $\mathscr{F} X \times \mathscr{F} X$ is supercomplete. Does it follow that $X$ is completely metrizable? More generally, let $X$ be a paracompact $p$-space such that $\mathscr{F} X \times \mathscr{F} X$ is supercomplete. Does it follow that $X$ is Čech-complete?

Remark 2. It was proved by Morita in [16] that if $X$ is a metrizable space such that $X \times Y$ is paracompact for every paracompact space $Y$, then $X$ is a countable union of closed locally compact subspaces.

Remark 3. Let $\mu X$ be a $C$-scattered supercomplete space. By Corollary 2.2. each finite power ${ }^{n}(\mu X)$ is supercomplete. It follows from [3] that the hyperspace $F(X)$ of all finite subsets of $X$ is paracompact. One can ask if $K(\mu X)$ is supercomplete. However, the space $X$ given in the proof of Theorem 1 in [18] is a $C$-scattered cosmic space such that $K(X)$ is not paracompact. Hence, $\mathscr{F} X$ is a $C$-scattered supercomplete space such that $K(\mathscr{F} X)$ is not supercomplete. Nevertheless, one can show that if $v Y$ is a Čech-complete supercomplete space, then so is $K(v Y)$, the proof being similar to that of 3.3.

Remark 4. Theorem 4.1. is not valid in the class of all paracompact spaces. Indeed, let $X$ be any paracompact space in which intersections of countable families of open sets are open. Then $\mathscr{F} X \times \mathscr{F} Y$ is supercomplete for any Lindelöf space $Y$.

Remark 5. Recently Miroslav Hušek and Jan Pelant have shown that $\lambda \Pi \mathscr{F} X_{i}=$ 
$=\mathscr{F} \Pi X_{i}$ for any family $\left\{X_{i}\right\}$ of paracompact Čech-complete spaces $X_{i}$. Their proof uses the fact the above equality holds for finite products, and this follows from Corollary 3.3.

Remark 6. A product $X \times Y$ of topological spaces $X$ and $Y$ is called rectangular if every finite normal cover of $X \times Y$ has a $\sigma$-locally finite refinement by cozerorectangles. Using the fact that every uniform cover has a $\sigma$-uniformly discrete refinement, it is easy to see that any product which has the $\lambda$-property is rectangular. By Remark 1 the converse is far from being true. It has been shown in [11] that a product $X \times Y$ of completely regular spaces $X$ and $Y$ is rectangular iff $\gamma(X \times Y)=\gamma X \times \gamma Y$ and $\gamma X \times \gamma Y$ is rectangular, where $\gamma$ denotes the topological (Dieudonné) completion. A similar statement is true for the $\lambda$-property. Indeed, suppose that $X \times Y$ has the $\lambda$-property. Then

$$
\begin{aligned}
& \lambda(\mathscr{F} \gamma X \times \mathscr{F} \gamma Y)=\lambda(\mathscr{F} \pi \mathscr{F} X \times \mathscr{F} \pi \mathscr{F} Y)=\lambda(\pi \mathscr{F} X \times \pi \mathscr{F} Y)= \\
= & \lambda \pi(\mathscr{F} X \times \mathscr{F} Y)=\pi \lambda(\mathscr{F} X \times \mathscr{F} Y)=\pi \mathscr{F}(X \times Y)=\mathscr{F} \gamma(X \times Y),
\end{aligned}
$$

were we have used the facts that $\pi \mathscr{F}=\mathscr{F} \pi \mathscr{F}$ and $\lambda \pi=\pi \lambda$. On the other hand, suppose that $\gamma(X \times Y)=\gamma X \times \gamma Y$ and $\gamma X \times \gamma Y$ has the $\lambda$-property. Then

$$
\begin{gathered}
\pi \lambda(\mathscr{F} X \times \mathscr{F} Y)=\lambda \pi(\mathscr{F} X \times \mathscr{F} Y)=\lambda(\pi \mathscr{F} X \times \pi \mathscr{F} Y)= \\
=\lambda(\mathscr{F} \gamma X \times \mathscr{F} \gamma Y)=\mathscr{F}(\gamma X \times \gamma Y)=\mathscr{F} \gamma(X \times Y)=\pi \mathscr{F}(X \times Y) .
\end{gathered}
$$

Since $\pi$ can be cancelled in any equation $\pi \mu S=\pi v S$, we have $\lambda(\mathscr{F} X \times \mathscr{F} Y)=$ $=\mathscr{F}(X \times Y)$, as required. One obtains as a corollary a uniform-theoretic proof of the result (of Pupier and Morita) that $\gamma(X \times K)=\gamma X \times K$ whenever $K$ is compact and $X$ is completely regular.

\section{References}

[1] Arhangel'skii, A.: On a class of spaces containing all metric and all locally bicompact spaces. Soviet Math. Dokl. 4, 1963, pp. 1051-1055.

[2] Artico, G., and R. Moresco: Notes on the topologies and uniformities of hyperspaces. Rend. Sem. Mat. Univ. Padova 63, 1980, pp. 51-60.

[3] Bell, M. G.: Hyperspaces of finite subsets. Topological structures, II (Proc. Sympos. Topology and Geom. Amsterdam), Part 1, Math. Centre Tracts 115, Math. Centrum, Amsterdam, 1979, pp. 15-28.

[4] Ferrier, J.: Paracompacite et espaces uniformes. Fund. Math. LX II. 1968, 7-30.

[5] Frolik, Z.: On the topological product of paracompact spaces. Bull. Acad. Pol. Sci. Math. 8 , 1960, pp. 747-750.

[6] Frolik, Z.: On paracompact uniform spaces. Czechoslovak Math. Journal 33 (108) 1983, pp. 476-484.

[7] Ginsburg, S., and J. R. Isbell: Some operators on uniform spaces. Trans. Amer. Math. Soc. 93, 1959, pp. 145-168.

[8] Hohti, A.: On uniform paracompactness. Math. Soc. 93, 1959, pp. 145-168. Ann. Acad. Scient. Fenn. Ser. A, I. Mathematica Dissertationes 36, 1981.

[9] Hohti, A.: On supercomplete uniform spaces. Proc. Amer. Math. Soc. 87: 3, 1983. 
[10] Hohti, A.: On uniform hyperspaces. Topology Proceedings 9, (1984), pp. 61-83.

[11] Hoshina, T. and $K$. Morita: On rectangular products of topological spaces. Topology and its applications 11,1980 , pp. 47-57.

[12] Isbell, J. R.: Supercomplete spaces. Pacific J. Math. 12, 1962, pp. 287-290.

[13] Isbell, J. R.: Uniform spaces. Mathematical Surveys No. 12, 1962, American Mathematical Society, Providence, Rhode Island, 1964.

[14] Isbell, J. R.: Atomless parts of spaces. Math. Scand. 31, 1972, pp. 5-32.

[15] Isbell, J. R.: Product spaces in locales. Proc. Amer. Math. Soc. 81: 1, 1981, pp. 116-118.

[16] Morita, K.: On the product of paracompact spaces. Proc. Japan Acad. 39, 1963, pp. 559 to 563.

[17] Morita, K.: Completion of hyperspaces of compact subsets and topological completion of open-closed maps. Gen. Top. Appl. 4, 1974, pp. 217-233.

[18] Okuyama, A.: Note on hyperspaces consisting of compact sets. Math. Japonica 24: 3, 1979, pp. 301-305.

[19] Shapiro, H.: Extensions of pseudometrics. Can. J. Math. 18, 1966, pp. 981-998.

[20] Telgársky, R.: $C$-scattered and paracompact spaces. Fund. Math. 73, 1971, pp. 59-74.

[21] Walker, R. C.: The Stone-Čech compactification. Ergebnisse der Mathematik und ihrer Grenzgebiete, Band 83, Springer-Verlag, Berlin-Heidelberg-New York, 1974.

[22] Zenor, P.: On the completeness of the space of compact subsets. Proc. Amer. Math. Soc. 26, 1970, pp. 190-192.

Author's address: University of Helsinki, Department of Mathematics, Hallituskatu 15, 00100 Helsinki 10, Finland. 